\section{MacoPharma THERAFLEX-Verfahren}

\section{Zulassung von Methylenblau- Plasma in Deutschland}

Das Paul-Ehrlich-Institut (PEI) hat am 15.1.2007 die erste Zulassung für die Herstellung und den Vertrieb von therapeutischem Einzelplasma erteilt, das nach dem THERAFLEX Methylenblau (MB)-Verfahren von MacoPharma virusinaktiviert wird. Bei dem pharmazeutischen Unternehmer bzw. der Herstellungsstätte handelt es sich um das Institut für Transfusionsmedizin der Universitätskliniken Essen (Prof. Dr. R. Moog). Das Arzneimittel erhält die Kennzeichnung «Gefrorenes Frischplasma MB (E)». Bei dem THERAFLEX Methylenblau-Plasma-Verfahren handelt es sich um eine Methode der photodynamischen Virusinaktivierung von Plasma, die zuerst vom DRK-Blutspendedienst Springe entwickelt wurde und in einer Kooperation zwischen Springe und

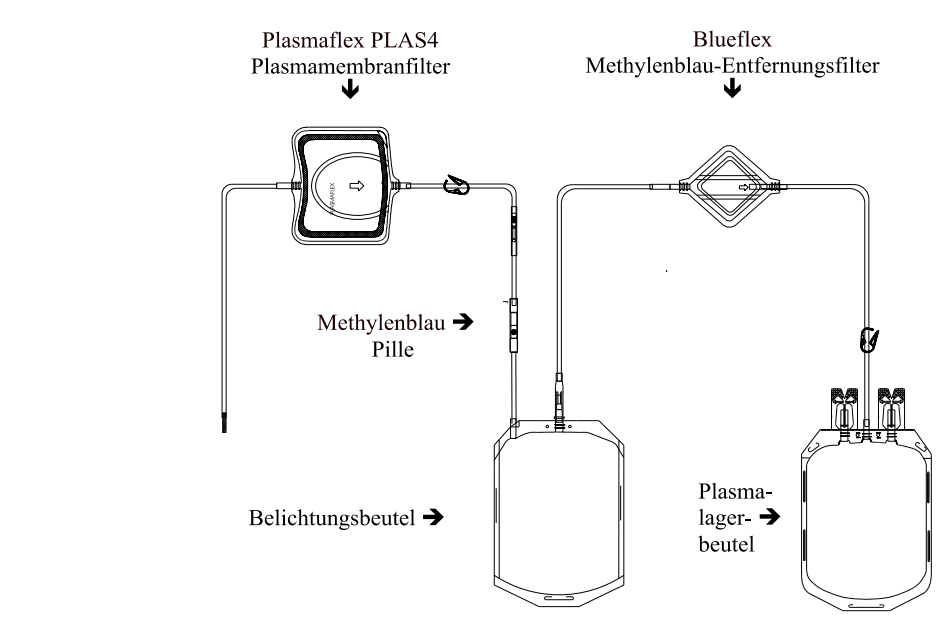

MacoPharma weiterentwickelt wurde. Im Plasma vorhandene Viren werden effektiv inaktiviert, so dass eine Infektion durch virale Erreger verhindert wird. Beim THERAFLEX-Verfahren wird das therapeutische Plasma zuerst einer Membranfiltration unterzogen, anschließend im geschlossenen System über eine MB-Pille $1 \mu \mathrm{M}$ MB zugegeben und $20 \mathrm{~min}$ mit einer Belichtungsmaschine «MACOTRONIC» mit sichtbarem Licht bestrahlt. Anschließend wird das MB und Photoprodukte durch einen speziellen Filter «BLUEFLEX» entfernt und so behandelte Plasma anschließend tiefgefroren.

Durch dieses Verfahren wird die Sicherheit bezüglich viraler Kontaminationen in Plasmen erhöht. Insbesondere neue, unbekannte Viren, für die keine Testverfahren vorliegen, können hierbei eliminiert werden.

Ein weiterer Vorteil liegt in der verbesserten Logistik, da eine Quarantänelagerung entfällt.

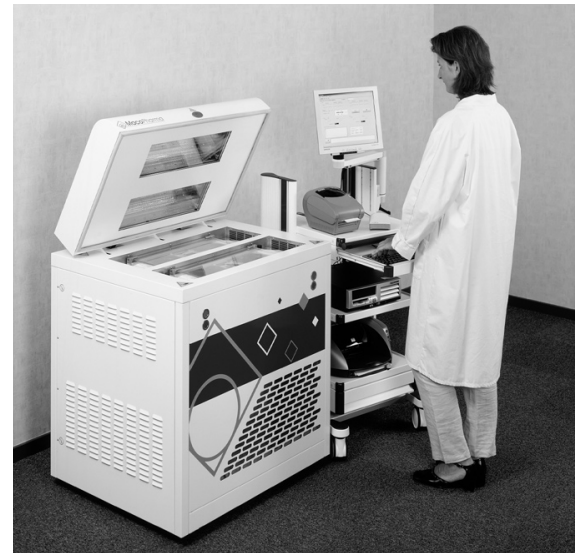

Abb. 2. Belichtungsmaschine Macotronic.

Das THERAFLEX MB-Verfahren wird bereits seit Jahren in verschiedenen europäischen Ländern eingesetzt, unter anderem in Spanien, Belgien, Italien, Großbritannien, Griechenland und Frankreich. Über 3 Millionen therapeutische MB-behandelte Einzelplasmen wurden mit ausgezeichneter Verträglichkeit alleine in Europa bereits klinisch eingesetzt. Die pharmazeutischen Eigenschaften, die Anwendungsgebiete sowie die Lagerbarkeit unterscheiden sich praktisch nicht von konventionellem therapeutischen Frischplasma.

Weitere Informationen bei MacoPharma International GmbH Dr. S. Reichenberg / Dr. W.H. Walker Robert-Bosch-Straße 11 63225 Langen, Deutschland Tel. +49 6103 9008-64, -24 stefan.reichenberg@macopharma.de wolfram.walker@macopharma.de

Abb. 1. Beutelsystem

\title{
Ticker+++ Ticker+++ Ticker+++ Ticker+++ Ticker+++ Ticker+++ Ticker+++
}

GlaxoSmithKline Deutschland hat zum 15. Januar 2007 die Preise für das synthetisch hergestellte Antikoagulans Fondaparinux-Natrium (Arixtra ${ }^{\circledR}$ ) für die meisten Packungsgrößen zwischen 14 und $54 \%$ gesenkt. Mit diesem Schritt leistet GSK einen aktiven Beitrag, damit der innovative Wirkstoff in Deutschland jetzt wirtschaftlich einsetzbar ist.

Weitere Informationen bei

GlaxoSmithKline GmbH und Co. KG

Dr. Anke Helten

Tel. +49 89 96044-8102, Fax -98102

anke.helten@gsk.com
Genzyme GmbH. Die kürzlich in der onlineAusgabe der internationalen Fachzeitschrift «Kidney International» vorab publizierten Ergebnisse der "Renagel in New Dialysis" (RIND)-Studie zeigen, dass Patienten, die mit Renage $^{\circledR}{ }^{\circledR}$ therapiert werden, eine signifikant geringere Mortalität aufweisen als Patienten, denen kalziumhaltige Phosphatbinder verabreicht wurden.

Weitere Informationen bei

Genzyme GmbH

Dr. Paul Fabry

Tel. +49 6102 3674-620, Fax -600

Paul.Fabry@Genzyme.com
Pharmion reicht die Anmeldung für die Genehmigung der Vermarktung von Thalidomid Pharmion ${ }^{\circledR}$ für die Behandlung von unbehandeltem multiplem Myelom in der Europäischen Union bei der European Medicines Agency ein. Ein Überlebensvorteil nach 21 Monaten wurde nachgewiesen, wenn Thalidomid zum Pflegestandard hinzugegeben wird. Die eingereichten Unterlagen basieren auf vier Phase-III-Studien mit mehr als 1400 Patienten.

Hill \& Knowlton Deutschland

Frank Vergien

Tel. +49 69 97362-35, Fax -39

frank.vergien@HillandKnowlton.com 